\title{
Recurrent Nephritis and/or Pulmonary Hemorrhage in Patients with Anti-Glomerular Basement Membrane Disease with and without ANCA Positivity
}

\author{
Nicole Droz ${ }^{\mathrm{a}}$ Alexis Katz ${ }^{\mathrm{a}}$ Aditi Patel $^{\mathrm{a}}$ Isaac Briskin ${ }^{\mathrm{b}}$ John Sedor ${ }^{\mathrm{c}}$ \\ Rula A. Hajj Alia \\ aOrthopedic and Rheumatologic Institute, Cleveland Clinic Foundation, Cleveland, OH, USA; ${ }^{b}$ Department of \\ Quantitative Health Sciences, Cleveland Clinic Foundation, Cleveland, OH, USA; 'Department of Nephrology, \\ Cleveland Clinic Foundation, Cleveland, $\mathrm{OH}$, USA
}

\section{Keywords}

Anti-GBM disease · Glomerulonephritis · Vasculitis ·

Anti-neutrophilic cytoplasmic antibodies

\begin{abstract}
Introduction: Anti-glomerular basement membrane (antiGBM) disease is characterized by rapidly progressive glomerular nephritis with or without pulmonary hemorrhage with disease severity correlating with antibody titer. Following treatment, relapse is rare but has been reported in the literature. The objective of this study was to assess for clinical, serologic, and histologic differences associated with disease relapse in patients with anti-GBM disease. Methods: Patients seen at our facility between 1997 and 2017 were screened for anti-GBM disease by ICD 9/10 codes. They were included if the diagnosis was confirmed by a board-certified rheumatologist or nephrologist and had positive antibodies and/or biopsy results consistent with anti-GBM disease. Relapsing disease was defined as recurrence of pulmonary or renal manifestations after achieving remission following the initial presentation. All charts were reviewed for baseline demographics, clinical manifestations, and antibody positivity and compared between groups. Results: 40 patients were con-
\end{abstract}

karger@karger.com www.karger.com/gdz

Karger $\stackrel{\text { ' }}{5}$
(C) 2021 The Author(s)

Published by S. Karger AG, Basel

This is an Open Access article licensed under the Creative Commons Attribution-NonCommercial-4.0 International License (CC BY-NC) (http://www.karger.com/Services/OpenAccessLicense), applicable to the online version of the article only. Usage and distribution for commercial purposes requires written permission. firmed as having anti-GBM disease. Mean follow-up from disease onset to the date of last follow-up was 56.2 months. 8 patients had relapsing disease and 32 patients had nonrelapsing disease. Baseline characteristics and clinical manifestations were similar between groups. Patients with relapsing disease had a high incidence of anti-neutrophilic cytoplasmic antibody (ANCA) co-positivity as compared to nonrelapsing patients ( 50 vs. $15.6 \%$, respectively, $p=0.059$ ), but this did not reach statistical significance. In patients with relapsing disease, only one had positive anti-GBM antibodies at time of relapse. Conclusions: In this study, patients with relapsing disease had a high incidence of ANCA co-positivity (50\%). In patients with newly diagnosed anti-GBM disease, ANCAs should be obtained to assess for the risk of relapse and to help guide long-term follow-up and treatment.

(C) 2021 The Author(s)

Published by S. Karger AG, Basel

\section{Introduction}

Anti-glomerular basement membrane (anti-GBM) disease is a relatively rare immune complex-mediated small vessel vasculitis with an estimated incidence of 1.64 per 1 million people per year [1]. The disease is character- 
ized by rapidly progressive glomerulonephritis with or without pulmonary hemorrhage and is mediated by pathogenic autoantibodies directed against the noncollagenous domain of the $\alpha 3$ chain of type 4 collagen. Antibodies are generally IgG, though IgA and IgM have also been reported [2]. Disease severity correlates with antibody titer at presentation [3].

The disease is generally monophasic in nature and is often fulminant at the time of onset with many patients presenting with respiratory and/or renal failure. After treatment and documentation of negative anti-GBM antibodies in circulation, relapse is rare. However, around $3 \%$ of patients may experience relapse after treatment of their disease [4]. The underlying pathogenesis of disease relapse remains unclear and may be related to environmental exposures such as tobacco use or hydrocarbon exposure, concomitant infection, coexistent anti-neutrophilic cytoplasmic antibodies (ANCA)-associated vasculitis, or membranous nephropathy [1]. The objective of this study was to assess for clinical, serologic, and histologic differences between patients with relapsing and nonrelapsing disease which may be associated with disease relapse.

\section{Materials and Methods}

\section{Study Design and Patients}

In this retrospective study, patients aged 17 or older seen in a single academic center between 1997 and 2017 were initially screened for inclusion into the study by ICD 9/10 codes for antiGBM disease or Goodpasture's syndrome (446.21 and M31.0). Patients were deemed eligible for inclusion if they had documentation by a board-certified rheumatologist or nephrologist at our institution confirming their diagnosis and had positive anti-GBM antibodies and/or biopsy results consistent with a diagnosis of anti-GBM disease. Serum anti-GBM antibodies were identified using conventional commercially available assays, which varied over time but included both ELISA and Western blot assays. Renal biopsies were considered diagnostic of anti-GBM disease if they demonstrated a crescentic glomerulonephritis with linear deposition of immunoglobulin along the basement membrane. Lung biopsies were considered positive if they showed evidence of pulmonary hemorrhage with accumulation of hemosiderin-laden macrophages in the alveolar spaces with positive immunofluorescence staining along the alveolar basement membranes. Relapsing disease was defined as recurrence of glomerulonephritis or pulmonary hemorrhage after achieving remission from the initial presentation and documentation of clearance of anti-GBM antibodies. The primary endpoint of this study was clinical, serologic, and histologic differences between patients with relapsing and nonrelapsing disease. All charts were reviewed for baseline characteristics, clinical manifestations of disease, renal histopathology, anti-GBM antibody, and ANCA positivity at the time of initial presentation and compared between those with relapsing and nonrelapsing dis- ease. Renal involvement was defined as rise in $\mathrm{Cr}$ above baseline, hematuria, RBC casts, or biopsy results confirming the disease. Pulmonary involvement was defined as hemoptysis, diffuse alveolar hemorrhage, or a pulmonary biopsy confirming the diagnosis. ANCA positivity was reported using either indirect immunofluorescence, ELISA, or bead-multiplex assays. Corresponding myeloperoxidase or proteinase-3 (PR3) antigens were also reported. The study protocol was reviewed and approved by the Institutional Review Board at the Cleveland Clinic Foundation.

\section{Data Collection}

Data were collected by retrospective chart review and were stored in a secure online database.

\section{Statistical Analysis}

Categorical measures were summarized with frequency (\%) and analyzed with Fisher's exact test. Continuous measures were summarized with mean (SD) or median (25th, 75th quartile) and analyzed with two-sample $t$ tests or Mann-Whitney $U$ tests depending on the normality of the data. Normality was verified by the Wilk-Shapiro test. Analyses consisted of comparing patients with relapsing disease to nonrelapsing patients. Analyses were performed using the "compareGroups" and "tidyverse" packages; $p$ values $<0.05$ were considered significant.

\section{Results}

164 patient charts were screened for inclusion. After the initial screening period, 40 patients were identified as having anti-GBM disease as confirmed by a rheumatologist or nephrologist at our institution. The main reason for exclusion was an alternative vasculitis diagnosis (such as ANCA-induced vasculitis or cryoglobulinemic vasculitis). Of the 40 patients identified as having anti-GBM disease, 25 patients had positive anti-GBM antibodies at the time of diagnosis. 17 patients had renal biopsies and 2 had a pulmonary biopsy for confirmation of their diagnosis. 32 patients had nonrelapsing disease, 8 had relapsing disease. Baseline demographics were similar between groups. There was near equal distribution of female and male sex between groups. The mean age of disease onset was 53.2 years for the nonrelapsing group and 46.9 years for the relapsing group. There was a high proportion of patients who were former or current smokers as compared to nonsmokers in both groups, but this was not different between groups (Table 1).

At initial presentation, both patient groups presented similarly. $93.8 \%$ of the patients with nonrelapsing disease and $100 \%$ of the patients with relapsing disease presented with renal manifestations. The mean peak $\mathrm{Cr}$ at diagnosis was higher in the nonrelapsing group $(7.24 \mathrm{~g} / \mathrm{dL})$ than the relapsing group $(5.37 \mathrm{~g} / \mathrm{dL})$, but this was not statistically significant. Renal histology was similar between 
Table 1. Disease characteristics and treatment at presentation

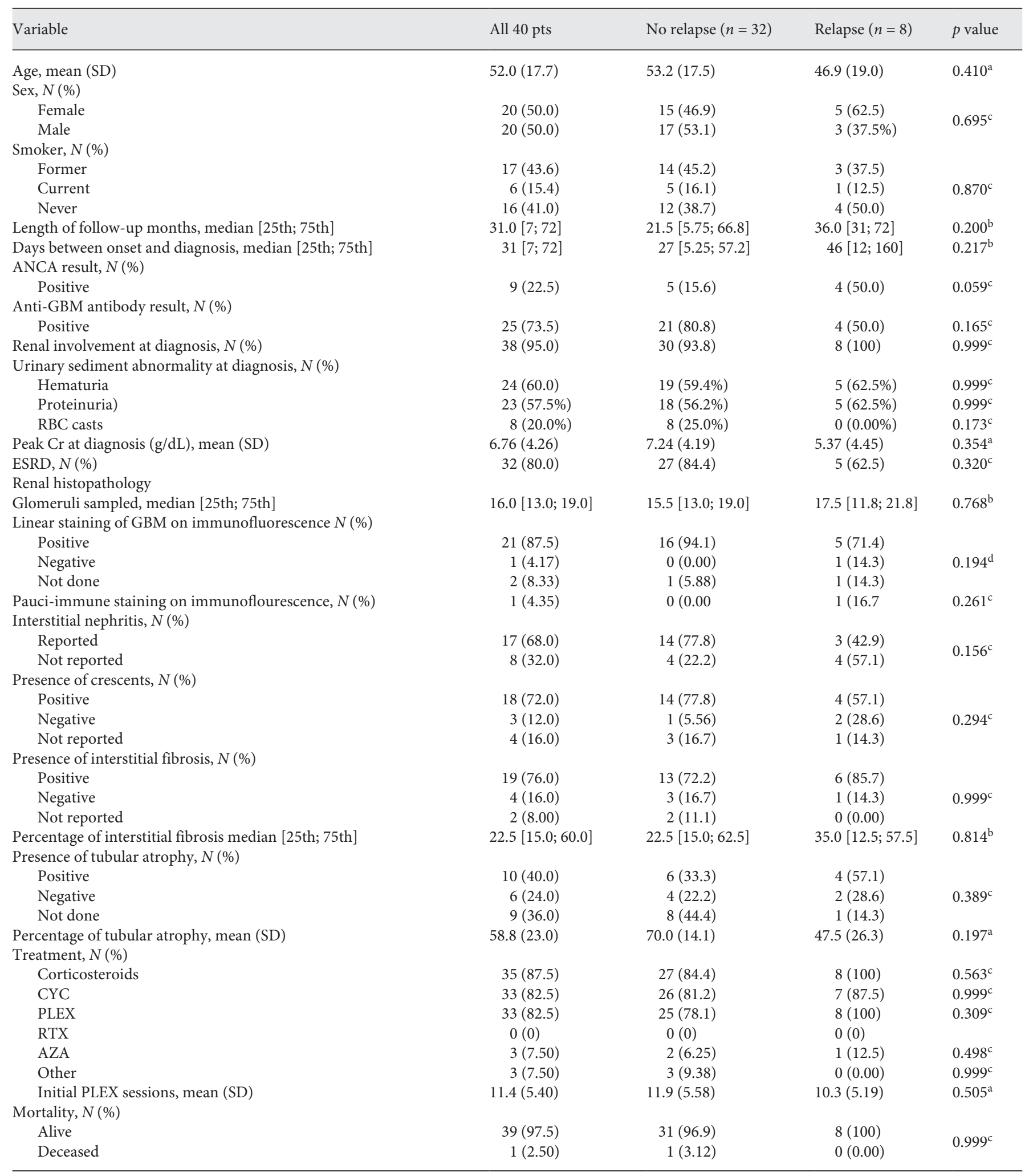

ANCA, anti-neutrophil cytoplasmic antibodies; GBM, glomerular basement membrane; $p$ values $<0.05$ were considered significant. ${ }^{\text {a }} 2$ sample $t$ test ${ }^{\mathrm{b}}$ Mann-Whitney U test. ${ }^{\mathrm{c}}$ Fisher's exact test. 
Table 2. ANCA and anti-GBM co-positive patients compared to anti-GBM positive patients alone

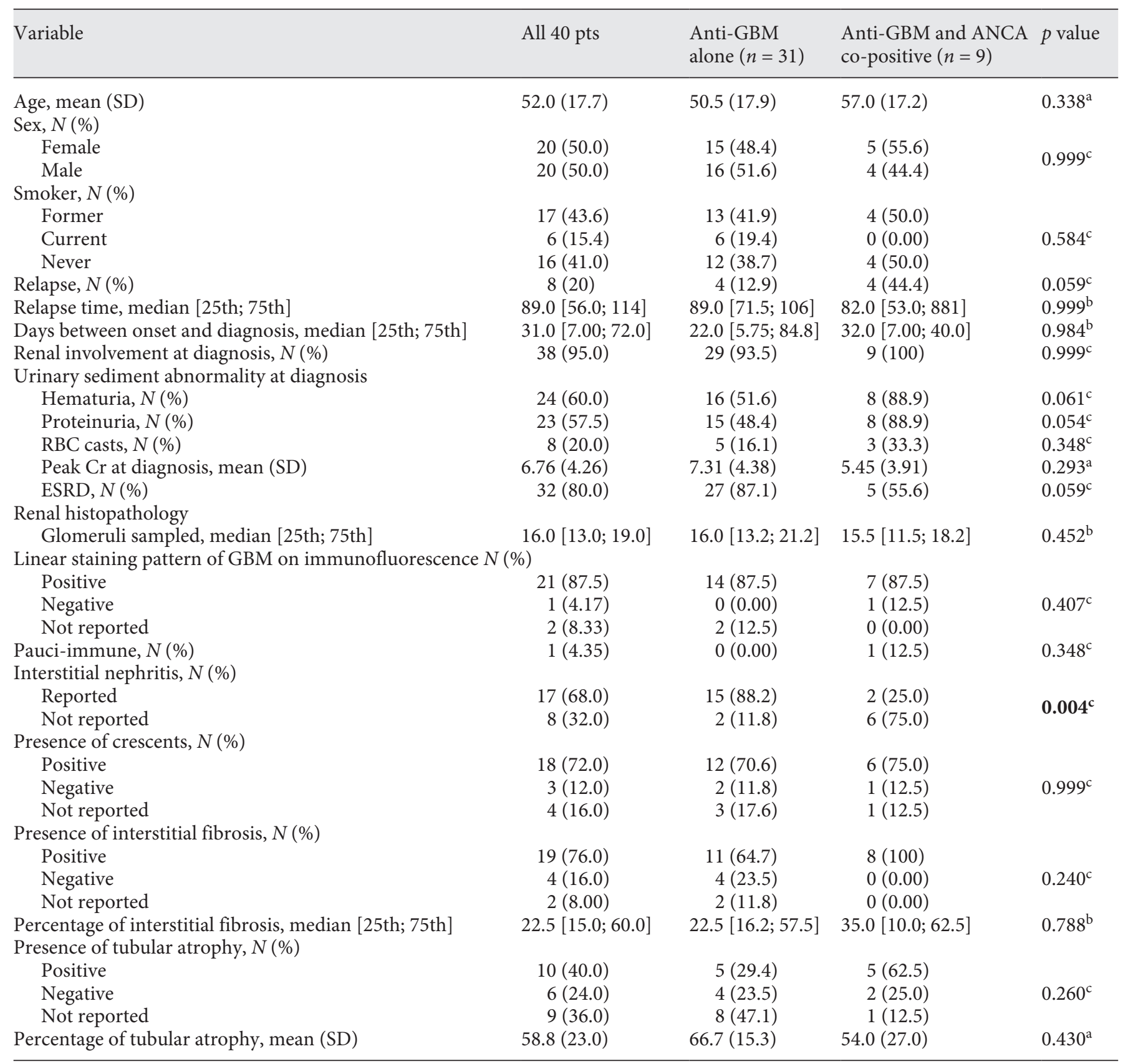

ANCA, anti-neutrophil cytoplasmic antibodies; GBM, glomerular basement membrane; $p$ values $<0.05$ were considered significant. a 2 sample $t$ test. ${ }^{\text {b }}$ Mann-Whitney $\mathrm{U}$ test. ${ }^{\mathrm{c}}$ Fisher's exact test.

groups with no differences when examining immunofluorescence patterns, interstitial fibrosis, tubular atrophy, or crescent formation (Table 1). Mortality was low, and only 1 patient fatality was recorded in the nonrelapsing group.
Pulmonary involvement at presentation was less frequent. Only $30 \%$ of the nonrelapsing patients and $37.5 \%$ of the relapsing patients presented with pulmonary disease at the time of presentation. 
Table 3. Comparison of baseline demographics and disease characteristics in patients who developed ESRD (dialysis dependent or transplantation) compared to those who have not

\begin{tabular}{|c|c|c|c|c|}
\hline Variable & All 40 pts & $\begin{array}{l}\text { Non-ESRD } \\
(n=8)\end{array}$ & $\operatorname{ESRD}(n=32)$ & $p$ value \\
\hline Age, mean (SD) & $52.0(17.7)$ & $54.4(15.4)$ & $51.3(18.4)$ & $0.642^{\mathrm{a}}$ \\
\hline \multicolumn{5}{|l|}{ Sex, $N(\%)$} \\
\hline Female & $20(50.0)$ & $4(50.0)$ & $16(50.0)$ & \multirow{2}{*}{$0.999^{c}$} \\
\hline Male & $20(50.0)$ & $4(50.0)$ & $16(50.0)$ & \\
\hline \multicolumn{5}{|l|}{ Smoker, $N(\%)$} \\
\hline Former & $17(43.6)$ & $3(37.5)$ & $14(45.2)$ & \multirow{3}{*}{$0.870^{c}$} \\
\hline Current & $6(15.4)$ & $1(12.5)$ & $5(16.1)$ & \\
\hline Never & $16(41.0)$ & $4(50.0)$ & $12(38.7)$ & \\
\hline Relapse time, median [25th; 75th] & $89.0[56.0 ; 114]$ & $106[97.5 ; 1,656]$ & $56.0[50.0 ; 74.0]$ & $0.157^{\mathrm{b}}$ \\
\hline Days between onset and diagnosis, median [25th; 75 th] & $31.0[7.00 ; 72.0]$ & $32.0[12.5 ; 62.0]$ & $26.0[7.00 ; 69.8]$ & $0.965^{\mathrm{b}}$ \\
\hline \multicolumn{5}{|l|}{ ANCA result, $N(\%)$} \\
\hline Negative & $2(18.2)$ & $1(20.0)$ & $1(16.7)$ & \multirow{2}{*}{$0.999^{c}$} \\
\hline Positive & $9(81.8)$ & $4(80.0)$ & $5(83.3)$ & \\
\hline \multicolumn{5}{|l|}{ Anti-GBM antibody result, $N(\%)$} \\
\hline Negative & $9(26.5)$ & $3(37.5)$ & $6(23.1)$ & \multirow{2}{*}{$0.649^{c}$} \\
\hline Positive & $25(73.5)$ & $5(62.5)$ & $20(76.9)$ & \\
\hline Renal involvement at diagnosis, $N(\%)$ & $38(95.0 \%)$ & $6(75.0 \%)$ & $32(100 \%)$ & $0.036^{\mathrm{c}}$ \\
\hline Urinary sediment abnormality at diagnosis-hematuria, $N(\%)$ & $24(60.0)$ & $6(75.0)$ & $18(56.2)$ & $0.439^{\mathrm{c}}$ \\
\hline Proteinuria, $N(\%)$ & $23(57.5)$ & $6(75.0)$ & $17(53.1)$ & $0.428^{\mathrm{c}}$ \\
\hline RBC casts, $N(\%)$ & $8(20.0)$ & $2(25.0)$ & $6(18.8)$ & $0.650^{\mathrm{c}}$ \\
\hline Peak $\mathrm{Cr}$ at diagnosis, mean (SD) & $6.76(4.26)$ & $2.07(1.28)$ & $8.10(3.83)$ & $<0.001^{\mathrm{a}}$ \\
\hline \multicolumn{5}{|l|}{ Renal histopathology } \\
\hline Glomeruli sampled, median [25th; 75th] & $16.0[13.0 ; 19.0]$ & $10.0[5.00 ; 18.0]$ & $16.0[14.0 ; 22.0]$ & 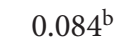 \\
\hline \multicolumn{5}{|c|}{ Linear staining pattern of GBM on immunofluorescence, $N(\%)$} \\
\hline Positive & $21(87.5)$ & $4(80.0)$ & $17(89.5)$ & \multirow{3}{*}{$0.240^{\mathrm{c}}$} \\
\hline Negative & $1(4.17)$ & $1(20.0)$ & $0(0.00)$ & \\
\hline Not reported & $2(8.33)$ & $0(0.00)$ & $2(10.5)$ & \\
\hline Pauci-immune, $N(\%)$ & $1(4.35)$ & $1(20.0)$ & $0(0.00)$ & $0.217^{\mathrm{c}}$ \\
\hline \multicolumn{5}{|l|}{ Interstitial nephritis, $N(\%)$} \\
\hline Reported & $17(68.0)$ & $2(40.0)$ & $15(75.0)$ & \multirow{2}{*}{$0.283^{c}$} \\
\hline Not reported & $8(32.0)$ & $3(60.0)$ & $5(25.0)$ & \\
\hline \multicolumn{5}{|l|}{ Presence of crescents, $N(\%)$} \\
\hline Positive & $18(72.0)$ & $2(40.0)$ & $16(80.0)$ & \multirow{3}{*}{$0.061^{\mathrm{c}}$} \\
\hline Negative & $3(12.0)$ & $2(40.0)$ & $1(5.00)$ & \\
\hline Not reported & $4(16.0)$ & $1(20.0)$ & $3(15.0)$ & \\
\hline \multicolumn{5}{|l|}{ Presence of interstitial fibrosis $N(\%)$} \\
\hline Positive & $19(76.0)$ & $4(80.0)$ & $15(75.0)$ & \multirow{3}{*}{$0.999^{c}$} \\
\hline Negative & $4(16.0)$ & $1(20.0)$ & $3(15.0)$ & \\
\hline Not reported & $2(8.00)$ & $0(0.00)$ & $2(10.0)$ & \\
\hline Percentage of interstitial fibrosis median [25th; 75 th] & $22.5[15.0 ; 60.0]$ & $55.0[50.0 ; 65.0]$ & $20.0[11.2 ; 51.2]$ & $0.087^{\mathrm{b}}$ \\
\hline \multicolumn{5}{|l|}{ Presence of tubular atrophy, $N(\%)$} \\
\hline Positive & $10(40.0)$ & $4(80.0)$ & $6(30.0)$ & \multirow{3}{*}{$0.128^{\mathrm{c}}$} \\
\hline Negative & $6(24.0)$ & $1(20.0)$ & $5(25.0)$ & \\
\hline Not reported & $9(36.0)$ & $0(0.00)$ & $9(45.0)$ & \\
\hline Percentage of tubular atrophy, mean (SD) & $58.8(23.0)$ & $60.0(14.1)$ & $57.5(32.0)$ & $0.893^{\mathrm{a}}$ \\
\hline
\end{tabular}

ANCA, anti-neutrophil cytoplasmic antibodies; GBM, glomerular basement membrane; $p$ values $<0.05$ were considered significant. a 2 sample $t$ test. ${ }^{b}$ Mann-Whitney $U$ test. ${ }^{c}$ Fisher's exact test. 
Table 4. Disease characteristics at the time of relapse

\begin{tabular}{lll}
\hline Variable & $\begin{array}{l}\text { Relapse } \\
(n=8)\end{array}$ & $N$ \\
\hline
\end{tabular}

\begin{tabular}{lcc} 
Relapse time (weeks), median [25th; 75th] & $89.0[56.0 ; 114]$ & 7 \\
\hline Serologies, N (\%) & $4(80.0)$ & 5 \\
ANCA positive at diagnosis & $3(75.0)$ & 4 \\
ANCA positive at relapse & $4(50.0)$ & 8 \\
Anti-GBM antibody positive at diagnosis & $1(20.0)$ & 5 \\
Anti-GBM antibody positive at relapse & $5(83.3)$ & 6 \\
Pulmonary involvement at relapse & $5(62.5)$ & 8 \\
Renal involvement at relapse & $3.05[2.93,3.20]$ & 8 \\
Peak Cr at relapse, median [25th; 75th]
\end{tabular}

Urinary sediment abnormality at relapse, $N$ (\%)

$\begin{array}{lll}\text { Hematuria } & 3(37.5) & 8 \\ \text { Proteinuria) } & 4(50.0) & 8 \\ \text { RBC casts } & 0(0) & 8\end{array}$

\begin{tabular}{lll}
\hline $\begin{array}{l}\text { Renal histopathology at relapse, } N(\%) \\
\text { Linear staining of GBM on }\end{array} \quad$ & \\
$\quad$ immunofluorescence & $2(66.7)$ & 3 \\
$\begin{array}{l}\text { Pauci-immune staining on } \\
\quad \text { immunoflourescence }\end{array}$ & $1(33.3)$ & 3 \\
Interstitial nephritis & $2(100)$ & 2 \\
Presence of crescents & $1(50.0)$ & 2 \\
Presence of interstitial fibrosis & $2(100)$ & 2 \\
Presence of tubular atrophy & $1(50.0)$ & 2
\end{tabular}

\begin{tabular}{lcc}
\hline Initial treatment, $N(\%)$ & & \\
Corticosteroids & $8(100)$ & 8 \\
CYC & $7(87.5)$ & 8 \\
PLEX & $8(100)$ & 8 \\
RTX & $0(0)$ & 8 \\
AZA & $1(12.5)$ & 8 \\
Initial PLEX sessions, mean (SD) & $10.3(5.19)$ & 7
\end{tabular}

\begin{tabular}{lcc}
\hline Treatment of relapse & & \\
Corticosteroids & $8(100)$ & 8 \\
CYC & $3(37.5)$ & 8 \\
PLEX & $6(75.0)$ & 8 \\
RTX & $4(50.0)$ & 8 \\
Other & $1(12.5)$ & 8 \\
PLEX at relapse, median [25th; 75th] & $6.00[4.50 ; 6.50]$ & 8 \\
\hline Renal outcomes & & \\
ESRD prior to relapse & $2(25)$ & 8 \\
ESRD at the time of last follow-up & $5(62.5)$ & 8
\end{tabular}

$N=$ number of patients tested; ANCA, anti-neutrophil cytoplasmic antibodies; GBM, glomerular basement membrane; CYC, cyclophosphamide; PLEX, plasma exchange; AZA, azathioprine; RTX, rituximab.

At diagnosis, $80.8 \%$ of patients in the nonrelapsing group-tested positive for anti-GBM antibodies as compared to $50 \%$ of patients with relapsing disease. This was not statistically significant (Table 1). There was a higher number of patients in the relapsing group who tested positive for ANCA serologies than patients with nonrelapsing disease (50\% as compared to $15.6 \%)$ which approached statistical significance $(p=0.059)$ The most common pattern was $\mathrm{pANCA} /$ myeloperoxidase positivity (Table 1).

When comparing patients who had ANCA co-positive disease to patients with anti-GBM disease alone, there was no significant difference in baseline demographics, clinical presentation, or renal survival. Histopathology was similar between groups with the exception of interstitial nephritis which was more frequently reported in the anti-GBM group alone (Table 2).

A higher proportion of patients in the nonrelapsing group required dialysis at presentation as compared to the relapsing group (84.4\% as compared to $62.5 \%$ ), but this was not statistically significant. Patients were followed for a median of 31 months. At the time of last follow-up, a high percentage of patients (32/40 or $80 \%)$ were either dialysis-dependent or underwent renal transplantation and considered to have ESRD. Patients with ESRD at time of last follow-up had a higher $\mathrm{Cr}$ at presentation as than patients who did not reach ESRD (Table 3).

Patients were most often treated with combination corticosteroids, cyclophosphamide, and plasma exchange. The mean number of plasma exchange sessions was 11.9 in the nonrelapsing group and 10.3 in the relapsing group (Table 1).

Of the 40 patients included in this study, 8 had a relapse of their disease. The median time to relapse was 89 weeks (range 6-480 weeks). Two patients had a relapse of disease after being dialysis dependent following their initial presentation. These patients' relapses were characterized by pulmonary involvement in the form of diffuse alveolar hemorrhage. There were no relapses after transplantation. At the time of relapse, $62.5 \%$ of patients presented with renal manifestations, and $83.3 \%$ presented with pulmonary manifestations of their disease. In patients who relapsed, 4 patients had positive anti-GBM antibodies at the time of initial presentation. After treatment, all patients who had positive anti-GBM antibodies had return of titers to within normal ranges. Only 1 patient had positive anti-GBM antibodies at the time of relapse. ANCA antibodies were not routinely recollected at the time of relapse but did remain positive in 3 of the 4 patients retested at the time of relapse. In patients who were re-biopsied, 2 patients demonstrated linear deposition of immunoglobulin staining along the GBM, and 1 patient had a pauci-immune staining on immunofluores- 
cence. All patients were initially treated with corticosteroids and plasma exchange, and 7 patients were treated with cyclophosphamide at initial presentation. 1 patient was treated with azathioprine maintenance immunosuppression prior to relapse. At the time of relapse, patients were again commonly treated with combination therapy with corticosteroids, an immunosuppressive agent, and plasma exchange. Cyclophosphamide was used to treat $37.5 \%$ of patients. Rituximab was used to treat $50 \%$ of patients, generally as salvage therapy after they had failed conventional treatment with cyclophosphamide (Table 4). In patients who relapsed, 2 were ESRD prior to relapse. After relapse 5 patients $(62.5 \%)$ had progressed to ESRD. There were no fatalities in the relapsing group.

\section{Discussion}

In this study, we compared clinical, serologic, and histologic findings in patients with relapsing and nonrelapsing anti-GBM disease. There were no differences in the baseline demographics, disease characteristics, or histopathology between the relapsing and nonrelapsing disease cohorts. Relapsing disease was common and was associated with a high incidence of ANCA co-positivity, though a larger study would be needed to evaluate for a statistically significant difference. Anti-GBM antibody positivity did not correlate with disease relapse.

It is well known that antibodies directed toward the alpha 3 chain of type 4 collagen are pathogenic. Lerner and colleagues elucidated this mechanism by inducing glomerulonephritis in squirrel monkeys after transfer of serum and renal eluate immunoglobulins from patients with anti-GBM disease [5]. In our study, we found a low proportion of patients who had anti-GBM positivity at presentation and at the time of relapse. At onset of disease, only $80.8 \%$ of nonrelapsing patients and $50 \%$ of relapsing patients tested positive for anti-GBM antibodies. At the time of relapse, only one patient was found to have anti-GBM positivity. Previous reports have suggested an incidence of antibody-negative anti-GBM disease to be around $16 \%$ of cases [6]. Patients in our study were retrospectively enrolled over a 20 -year time frame, and different assays were utilized to detect circulating anti-GBM antibodies (by ELISA or indirect immunofluorescence assays); however, the commercially available assays varied with time. ELISA assays have varied sensitivity and specificity and may have impacted the proportion of antiGBM positive patients [7]. Additionally, most conventional ELISA assays detect IgG1 anti-GBM antibodies but other subclasses (such as IgG4) may not be readily detected on commercially available tests [8]. Indirect immunofluorescence is even less sensitive at detecting antiGBM antibodies than ELISA testing [9]. It is not clear at this point whether the low prevalence of anti-GBM antibodies is related to assay variability or other reasons.

Historically, relapse of anti-GBM disease was felt to be rare and was limited to case series [10-13]. In our study, $20 \%$ of patients had a relapse of their disease. This is higher than what was published previously in the literature. One possible explanation is the high incidence of ANCA co-positivity seen in the relapsing cohort. Further, we may have captured a sicker patient population given the referral bias to our tertiary center. In a large cohort of patients studied by McAdoo et al. [14] authors found that patients with ANCA and GBM co-positive disease had a relapse rate of $22 \%$ early in the disease course and approximately $50 \%$ had recurrence during long-term follow-up, similar to the rates of relapse seen with ANCAassociated vasculitis. There were no disease relapses in patients with positive anti-GBM disease alone (without positive ANCA). Authors hypothesized that patients who are ANCA and GBM co-positive may have an intermediate phenotype with severe renal disease and high frequency of lung hemorrhage at the time of initial presentation but also with continued risk of relapse through follow-up. Given the high rate of ANCA co-positivity seen in the relapsing cohort, it is possible the ANCA positivity may confer the risk of relapse. Previous reports have demonstrated a rise in ANCA titer or seroconversion from ANCA negative to ANCA positive prior to relapse [14]. In our cohort, ANCAs were not consistently checked at the time of disease relapse but did remain positive in 3 of 4 patients who were originally checked at disease onset.

In conclusion, our study has demonstrated patients with relapsing and nonrelapsing diseases have similar characteristics, but patients with relapsing disease had a high incidence of ANCA positivity consistent with previous reports. This did not achieve statistical significance, but interpretation is limited by the small sample size. Given our findings and previous reports, we recommend testing for ANCAs in patients with newly diagnosed antiGBM disease, to assess for the risk of relapse and to help guide long-term follow-up and treatment. In patients who are ANCA and anti-GBM antibody co-positive, we recommend routine follow-up and laboratory monitoring for evidence of disease recurrence and consideration for maintenance immunosuppressive therapy. Larger studies are needed to validate our results. 


\section{Statement of Ethics}

This study protocol was reviewed and approved by the Institutional Review Board at the Cleveland Clinic Foundation IRB \# 17 655. This study was considered minimal risk and was conducted via retrospective chart review using data collected for routine clinical purposes. A waiver of informed consent and waiver of HIPAA authorization were approved through our IRB given the study design and minimal risk.

\section{Conflict of Interest Statement}

The authors have no conflicts of interest to declare.

\section{Funding Sources}

There was no funding for this project.

\section{Author Contributions}

Nicole Droz, MD:

1. Conception or design, or analysis and interpretation of data, or both.

2. Drafting the article or revising it.

3. Providing intellectual content of critical importance to the work described.

4. Final approval of the version to be published.
Alexis Katz, DO:

1. Conception or design, or analysis and interpretation of data, or both.

2. Drafting the article or revising it.

3. Providing intellectual content of critical importance to the work described.

4. Final approval of the version to be published. Aditi Patel, MD:

1. Conception or design, or analysis and interpretation of data, or both.

2. Drafting the article or revising it.

3. Providing intellectual content of critical importance to the work described.

4. Final approval of the version to be published. Isaac Briskin, MA:

1. Conception or design, or analysis and interpretation of data, or both.

2. Drafting the article or revising it.

3. Providing intellectual content of critical importance to the work described.

4. Final approval of the version to be published. John Sedor, MD:

1. Conception or design, or analysis and interpretation of data, or both.

2. Drafting the article or revising it.

3. Providing intellectual content of critical importance to the work described.

4. Final approval of the version to be published. Rula Hajj Ali, MD:

1. Conception or design, or analysis and interpretation of data, or both.

2. Drafting the article or revising it.

3. Providing intellectual content of critical importance to the work described.

4. Final approval of the version to be published.

\section{References}

1 Canney M, O’Hara PV, McEvoy CM, Medani S, Connaughton DM, Abdalla AA, et al. Spatial and temporal clustering of anti-glomerular basement membrane disease. Clin J Am Soc Nephrol. 2016;11(8):1392-9.

2 Salama AD, Levy JB, Lightstone L, Pusey CD. Goodpasture's disease. Lancet. 2001; 358(9285):917-20.

3 Segelmark M, Hellmark T, Wieslander J. The prognostic significance in Goodpasture's disease of specificity, titre and affinity of antiglomerular-basement-membrane antibodies. Nephron Clin Pract. 2003;94(3):c59-68.

4 Levy JB, Turner AN, Rees AJ, Pusey CD. Long-term outcome of anti-glomerular basement membrane antibody disease treated with plasma exchange and immunosuppression. Ann Intern Med. 2001;134(11):1033-42.

5 Lerner RA, Glassock RJ, Dixon FJ. The role of anti-glomerular basement membrane antibody in the pathogenesis of human glomerulonephritis. J Exp Med. 1967;126(6):9891004 .

Disease Recurrence in Anti-GBM Disease with and without ANCA
6 Fischer EG, Lager DJ. Anti-glomerular basement membrane glomerulonephritis: a morphologic study of 80 cases. Am J Clin Pathol. 2006 Mar;125(3):445-50.

7 Sinico RA, Radice A, Corace C, Sabadini E, Bollini B. Anti-glomerular basement membrane antibodies in the diagnosis of Goodpasture syndrome: a comparison of different assays. Nephrol Dial Transplant. 2006;21(2): 397-401.

8 Ohlsson S, Herlitz H, Lundberg S, Selga D, Mölne J, Wieslander J, et al. Circulating antiglomerular basement membrane antibodies with predominance of subclass IgG4 and false-negative immunoassay test results in anti-glomerular basement membrane disease. Am J Kidney Dis. 2014;63(2):289-93.

9 Wilson CB, Dixon FJ. Diagnosis of immunopathologic renal disease. Kidney Int. 1974; 5(6):389-401.

10 Rees AJ, Lockwood CM, Peters DK. Enhanced allergic tissue injury in Goodpasture's syndrome by intercurrent bacterial infection. Br Med J. 1977;2(6089):723-6.
11 Segelmark M, Dahlberg P, Wieslander J. AntiGBM disease with a mild relapsing course and low levels of anti-GBM autoantibodies. Clin Kidney J. 2012;5(6):549-51.

12 Liu P, Waheed S, Boujelbane L, Maursetter LJ Multiple recurrences of anti-glomerular basement membrane disease with variable antibody detection: can the laboratory be trusted? Clin Kidney J. 2016;9(5):657-60.

$13 \mathrm{Gu}$ B, Magil AB, Barbour SJ. Frequently relapsing anti-glomerular basement membrane antibody disease with changing clinical phenotype and antibody characteristics over time. Clin Kidney J. 2016;9(5):661-4.

14 McAdoo SP, Tanna A, Hrušková Z, Holm L, Weiner M, Arulkumaran N, et al. Patients double-seropositive for ANCA and antiGBM antibodies have varied renal survival, frequency of relapse, and outcomes compared to single-seropositive patients. Kidney Int. 2017;92(3):693-702. 